\title{
A Method of investigating the Geometry of Families of Curves, with Examples.
}

\author{
By George D. Valentine, M.A.
}

(Read 9th December 1910. Received 19th January 1911).

$\$ 1$. Consider the equation $f(\xi, \eta, x, y)=0$. If definite values of $\xi$ and $\eta$ be taken, and $x$ and $y$ be current co-ordinates, $f(\xi, \eta, x, y)=0$ is represented by a curve in the plane of $x y$. If all the possible values of $\xi$ and $\eta$ be taken in turn, $f(\xi, \eta, x, y)=0$ will be represented by a doubly infinite family of curves in the $x y$ plane. Similarly if all possible definite values of $x$ and $y$ be taken in turn and $\xi$ and $\eta$ be regarded as current co-ordinates, $f(\xi, \eta, x, y)=0$ will be represented by a doubly infinite family of curves in the $\xi \eta$ plane. Since the first and the second doubly infinite families of curves represent the same equation, it may be expected that some geometrical correspondence exists between them. It is the object of the present paper to investigate this correspondence.

$\$ 2$. We shall call curves in the $x y$ plane whose equation is of the form $f(\xi, \eta, x, y)=0$ where $\xi$ and $\eta$ have definite values, curves of the first family, and curves in the $\xi \eta$ plane whose equation is of the same form, but where it is now $x$ and $y$ that have definite values, curves of the second family. In the $\xi \eta$ plane any definite values of $\xi, \eta$ give a point uniquely determined. In the $x y$ plane the same values of $\xi \eta$ give a curve of the first family. The point $\xi, \eta$ in $\xi \eta$ plane may be said to correspond to the curve of the first family in the $x y$ plane, and vice versa. In some cases we may have $f(\xi, \eta, x, y) \equiv f\left(\xi^{\prime}, \eta^{\prime}, x, y\right)$ for certain values of $\xi, \eta$ and $\xi^{\prime}, \eta^{\prime}$. In such cases the points $\xi, \eta$ and $\xi^{\prime}, \eta^{\prime}$ will correspond to the same curve of the first family. We may term the points $\xi, \eta$ and $\xi^{\prime}, \eta^{\prime}$ associated points. All points associated with each other form a group, and we may speak of that group as a group corresponding to a curve of the first family. It will be observed that if a curve of the second family pass through one point of a group in its plane 
it will pass through all of them, for if $f(\xi, \eta, x, y)=0$ for certain values of $x$ and $y, f\left(\xi, \eta^{\prime}, x, y\right) \equiv f(\xi, \eta, x, y)=0$ for the same values. Where groups of associated points exist in the $\xi \eta$ plane, that plane may generally be divided into regions such that each region contains one point and one point alone of each group ; each such region may be regarded as corresponding to the whole of the $x y$ plane. Similarly a curve of the second family in the $\xi \eta$ plane corresponds to a point or group of associated points in the $x y$ plane.

§3. If any point be taken on a curve of the first family, the curve of the second family corresponding to that point passes through the point or group of points corresponding to the curve of the first family, and conversely.

For the condition that the point $x_{1}, y_{1}$ lies on the curve

is

$$
\begin{aligned}
& f\left(\xi_{1}, \eta_{1}, x, y\right)=0 \\
& f\left(\xi_{1}, \eta_{1}, x_{1}, y_{1}\right)=0
\end{aligned}
$$

and this is also the condition that the point $\xi_{1}, \eta_{1}$ lies on the curve $f\left(\xi, \eta, x_{1}, y_{1}\right)=0$ in the $\xi, \eta$ plane.

Corollary.-All the curves of the second family corresponding to points lying on a curve of the first family are concurrent at the point or group of points corresponding in the $\xi \eta$ plane to the given curve of the first family, and conversely all the points corresponding to curves of the first family lie upon the same curve of the second family.

\$4. The point or points of intersection of two curves of the first family correspond to the curve of the second family joining two points corresponding to the given curves of the first family, and conversely.

Corollary.-If two curves of a family cut at more than one point, their points of intersection form an associated group.

$\S 5$. If $\phi(x, y)=0$ be the envelope of the curves of the first family such that $\quad \psi(\xi, \eta)=0$, then $\quad \psi(\hat{\xi}, \eta)=0$ is the envelope of the curves of the second family

such that

$$
\begin{aligned}
& f(\xi, \eta, x, y)=0, \\
& \phi(x, y)=0,
\end{aligned}
$$


and $\psi(\xi, \eta)=0$ may be said to be the curve corresponding to the curve $\phi(x, y)=0$.

For let $\xi, \eta ; \xi+d \xi, \eta+d \eta$ be two consecutive values of $\xi$ and $\eta$ satisfying the equation $\psi(\xi, \eta)=0$.

Then

$$
\begin{aligned}
& f(\xi, \eta, x, y)=0 \\
& f(\xi+d \xi, \eta+d \eta, x, y)=0
\end{aligned}
$$

will be two consecutive curves in the $x y$ plane whose point of intersection lies on $\phi(x, y)=0$. Also the point of intersection of these two curves corresponds to the curve of the second family passing through $\xi, \eta$ and $\xi+d \xi, \eta+d \eta$, that is to the curve of the second family touching $\psi(\xi, \eta)=0$ at $\xi$, $\eta$.

Hence $\psi(\xi, \eta)=0$ is the envelope of the curves of the second family corresponding to points on $\phi(x, y)=0$, that is to curves of the second family for which $x$ and $y$ satisfy the relation. In this sense the curves $\phi(x, y)=0$ and $\psi(\xi, \eta)=0$ correspond.

$\S 6$. The simplest example of an equation of the form

is

$$
\begin{aligned}
& f(\xi, \eta, x, y)=0 \\
& \xi x+\eta y=1 .
\end{aligned}
$$

The application of the method to this equation gives us the theory of poles and polars.

As a more general example take

$$
\xi x^{n}+\eta y^{n}=1
$$

of which the equation last mentioned is a particular case.

We have on the $x y$ plane a curve of the $n^{\text {th }}$ degree. When $\xi$ is infinitely great, this curve degenerates to the axis of $y$; when $\eta$ is infinitely great to the axis of $x$. When both $\xi$ and $\eta$ are infinitely great it degenerates to a series of straight lines passing through the origin. If $(-1)^{n}=1$ these lines are all imaginary if $\xi$ and $\eta$ have the same sign and include a real pair if $\xi$ and $\eta$ have opposite signs. If $(-1)^{n}=-1$ these lines in every case include one real line. As it is our intention to confine ourselves to purely geometrical properties, we may neglect (in general) imaginary lines.

\$7. To a point in the $\xi \eta$ plane there corresponds a curve in the $x y$ plane, and to a point in the $x y$ plane a straight line in the $\xi \eta$ plane. 
If $(-1)^{n}=-1$ there are no real associated points in the $x y$ plane.

If $(-1)^{n}=1$ the points $x, y ;-x, y ; x,-y ;-x,-y$ form an associated group.

There are no associated points in the $\xi \eta$ plane.

$\$ 8$. The points on the straight line $x / a=y / b$ correspond to the system of parallel lines $\xi a^{n}+\eta b^{n}=k$ in the $\xi \eta$ plane, and the line $x / a=y / b$ itself corresponds to the point at infinity at which these parallels meet.

\$9. If $\mathbf{P}$ and $\mathrm{P}^{\prime}$ are two points on the $x y$ plane such that POP' is a right angle, and if $(-1)^{n}=-1$, the lines corresponding to $\mathrm{P}$ and $\mathrm{P}^{\prime}$ in the $\xi \eta$ plane are at right angles and conversely. If, however, $(-1)^{n}=1$, the lines corresponding to $\mathbf{P}$ and $\mathbf{P}^{\prime}$ in the $\xi \eta$ plane are not at right angles, but are such that each of them is at right angles to a line drawn to make the same angle with the axes as the other.

$$
\begin{array}{ll}
\$ 10 . \text { If } & \xi_{1} x^{n}+\eta_{1} y^{n}=1 \\
\text { and } & \xi_{2} x^{n}+\eta_{2} y^{n}=1
\end{array}
$$

be two curves in the $x y$ plane, we shall define the curve

$$
\left(\xi_{1}+\xi_{2}\right) x^{n}+\left(\eta_{1}+\eta_{2}\right) y^{n}=2
$$

as being the harmonic mean curve between them. It will be observed that the tangents to two curves and to their harmonic mean at the point of intersection, together with the radius vector at that point, form a barmonic pencil. If $n=1$, the curves degenerate into these tangents themselves.

Further, if any radius vector be drawn, and the intercepts on it by the two given curves be $r_{1}$ and $r_{2}$ and that by their harmonic mean curve $r_{3}$, then $r_{1}{ }^{n}, r_{3}{ }^{n}$, and $r_{2}{ }^{n}$ are in harmonic progression.

The points corresponding to the curves

and

$$
\begin{aligned}
& \xi_{1} x^{n}+\eta_{1} y^{n}=1 \\
& \xi_{2} x^{n}+\eta_{2} y^{n}=1
\end{aligned}
$$

are respectively $\xi_{1}, \eta_{1} ; \xi_{2}, \eta_{2} ;$ and $\frac{1}{2}\left(\xi_{1}+\xi_{2}\right), \frac{1}{2}\left(\eta_{1}+\eta_{2}\right)$, the last of which is the point bisecting the line joining the first two.

If we call these three points $P_{1}, P_{2}$ and $P_{3}$ respectively, and if $Q$ be any other point. Then if $Q R$ be drawn parallel to the line 
$\mathrm{P}_{1} \mathrm{P}_{2}, \mathrm{Q}\left\{\mathrm{P}_{1} \mathrm{P}_{3} \mathrm{P}_{2} \mathrm{R}\right\}$ is a harmonic pencil in the $\xi \eta$ plane; and to this there corresponds in the $x y$ plane four points all lying on the curve of the family corresponding to $Q$. These four points are its points of intersection with the curves (1), (3), (2), and with the radius vector through their point of intersection. Four points in the $x y$ plane so determined may be called a harmonic range.

Corollary.-When the equation is

$$
\xi x+\eta y=1
$$

we have the proposition-the poles of a harmonic pencil form a harmonic range, and conversely.

$\$ 11$. It will be convenient to find what is in the $x y$ plane the curve corresponding to the circle $\xi^{2}+\eta^{2}=r^{2}$.

This curve is the envelope of $\xi x^{n}+\eta y^{n}=1$ under the given condition.

Hence

$$
\begin{aligned}
& \frac{d \eta}{d \xi}=-\frac{x^{n}}{y^{n}}=-\frac{\xi}{\eta} \\
& \frac{\xi}{x^{n}}=\frac{\eta}{y^{n}}=\frac{1}{x^{2 n}+y^{2 n}} \\
& x^{2 n}+y^{2 n}=r^{2}\left(x^{2 n}+y^{2 n}\right)^{2} \\
& x^{2 n}+y^{2 n}=r^{-2} .
\end{aligned}
$$

We shall call a curve of the form $x^{2 n}+y^{2 n}=k^{2 n}$ an $n$-ic circle.

$\$ 12$. We are now in a position to write down a number of properties in the geometry of curves of the form $\xi x^{n}+\eta y^{\prime \prime}=1$.

Perhaps the simplest are those which relate to perspective properties.

We shall use the terms curvilinear triangle, curvilinear polygon, etc., to denote a triangle, polygon, etc., each of whose sides is formed by an arc of a curve of a family $\xi x^{n}+\eta y^{n}=1$.

The leading proposition in the perspective of rectilinear triangles is as follows, -if two triangles be such that the lines joining corresponding vertices are concurrent, the points of intersection of the corresponding sides are collinear, and conversely. Let us take triangles in the $\xi \eta$ plane possessing this property, and consider the corresponding figure in the $x y$ plane. To each triangle in the $\xi \eta$ plane corresponds a curvilinear triangle in the $x y$ plane, its vertices corresponding to the sides of the $\xi \eta$ triangle and its sides to the vertices of the latter. To the lines joining corresponding 
vertices of the $\xi \eta$ triangle correspond the points of intersection of corresponding sides of the $x y$ triangle, and to the point in which these lines are concurrent in the $\xi \eta$ plane corresponds a curve of the first family in the $x y$ plane passing through all the points of intersection of corresponding sides of the curvilinear triangles. Further, to the points of intersection of corresponding sides of the $\xi \eta$ triangle correspond the curves of the first family joining corresponding vertices of the curvilinear triangles, and all these curves must pass through the point in the $x y$ plane which corresponds to the straight line in the $\xi \eta$ plane in which the points of intersection of the corresponding sides are collinear.

Hence we have the following proposition-if two curvilinear triangles be such that the points of intersection of the corresponding sides lie on a curve of the family, the curves joining their corresponding vertices are concurrent, and conversely. That is to say, curvilinear triangles whose sides are arcs of curves of the form $\xi x^{n}+\eta y^{n}=1$ may possess the perspective property. We may generalise this proposition by saying that it is true of all curves of the form

$$
\xi \phi_{1}(x, y)+\eta \phi_{2}(x, y)+\phi_{3}(x, y)=0 \text {. }
$$

The proof is identical. The property appears to be characteristic of curves which are linear in two parameters, and to be true of no others.

Generally all propositions regarding rectilinear triangles which depend entirely on their perspective properties are true also of such curvilinear triangles.

For example, when three curvilinear triangles are two by two in perspective and have the same curve of the family as their axis of perspective, their three centres of perspective lie on a curve of the family, and when they have the same centre of perspective, their axes of perspective are concurrent.

$\$ 13$. Another proposition which may easily be generalised is Pascal's Theorem with the associated Theorem of Brianchon.

We know that if a rectilinear hexagon be inscribed in a circle, the intersections of the opposite sides, viz. the first and the fourth, the second and the fifth, and the third and the sixth are collinear, and if a hexagon be described about a circle, the three lines joining the opposite angular points are concurrent. 
Let the circle be a circle in the $\xi \eta$ plane of unit radius, and having its centre at the origin. There corresponds to it in the $x y$ plane the $n$-ic circle $x^{2 n}+y^{2 n}=1$.

To the six angular points of the inseribed hexagon correspond six curves of the first family touching the $n$-ic circle, and to its six sides the six points of intersection of consecutive tangent curves. If pairs of tangent curves intersect at more than one point, the proposition which will be deduced is true, whichever of the points is taken (they will then form an associated group). To the intersections of the sides of the rectilinear hexagon correspond the curvilinear diameters of the curvilinear hexagon, and since the intersections of the opposite sides of the rectilinear hexagon are collinear, the curvilinear diameters are concurrent. Similarly to the six sides of the hexagon escribed to the $\xi \eta$ circle correspond six points on the $n$-ic circle, to its six angular points, the six curves (properly chosen) joining these points, to its three diameters, the three points of intersection of the first and fourth, second and fifth. and third and sixth of these curves, all of which must lie on a curve of the family, since the diameters in the $\xi \eta$ plane are concurrent.

Hence Pascal's and Brianchon's Theorems are true of the curvilinear hexagon inscribed in and escribed to the $n$-ic circle.

\section{\$14. I shall now give a few properties of harmonic mean curves.}

Take the proposition in rectilinear geometry that the line joining the middle points of the sides of a triangle is parallel to the base.

Let $\mathrm{ABC}$ be a rectilinear triangle in the $\xi \eta$ plane, and $\mathrm{DE}$ the line joining the middle points of its sides. Then in the $x y$ plane (Fig. 1)

there corresponds to the line $\mathrm{AB}$ the point $\gamma$;
to the line $\mathrm{BC}$ the point $a$;
to the line $\mathrm{CA}$ the point $\beta$;
to the point $\mathrm{A}$ the curve $\beta \gamma$ :
to the point $\mathrm{B}$ the curve $\gamma \alpha$;
to the point $\mathrm{C}$ the curve $a \beta$;
to the point $\mathrm{D}$ the curve $\gamma \kappa$;


which is the harmonic mean curve between $\gamma \alpha$ and $\gamma \beta$;

to the point $\mathrm{E}$ the curve $\beta \kappa$, which is the harmonic mean curve between $\beta \gamma$ and $\beta a$;

to the line $\mathrm{DE}$ the point $\kappa$;

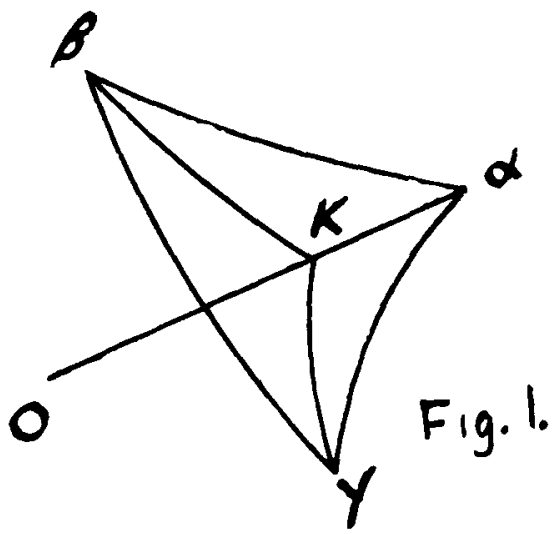

and since $\mathrm{DE}$ is parallel to $\mathrm{BC}$, therefore $\kappa$ and $a$ lie on the same straight line through the origin $O$ if $(-1)^{n}=-1$, and either on the same straight line through $O$, or on straight lines through $O$, making equal angles with the axes if $(-1)^{n}=1$; in the latter case there will in fact be four points corresponding to each of the lines BC, etc., which four points will form an associated group symmetrical about the origin, that is to say, each of the points $a, \beta, \gamma$ will have associated with it three points $a^{\prime}, a^{\prime \prime}, a^{\prime \prime \prime} ; \beta^{\prime}, \beta^{\prime \prime}, \beta^{\prime \prime \prime} ; \gamma^{\prime}, \gamma^{\prime \prime}, \gamma^{\prime \prime \prime}$; the harmonic mean curve $\gamma \kappa$ will pass through each of the points $\gamma^{\prime}, \gamma^{\prime \prime}, \gamma^{\prime \prime \prime}$; there will be three points $\kappa^{\prime}, \kappa^{\prime \prime}, \kappa^{\prime \prime \prime}$ associated with $\kappa$; the points $\kappa, \kappa^{\prime}, \kappa^{\prime \prime}, \kappa^{\prime \prime \prime}$ will lie two by two on two straight lines through the origin, making equal angles with the axes, and $\alpha, a^{\prime}, a^{\prime \prime} ; \alpha^{\prime \prime \prime}$ will also be two by two on the same straight lines. Having in this case shown at length the way in which the presence of associated points modifies the proposition, we shall not usually think it necessary to consider them in future, but shall state the propositions generally in the form which they take when there are no associated points. The form which a proposition takes when there are associated points with equations of the type $\xi x^{n}+\eta y^{n}=1$ can be at once deduced by observing that in that case there will be symmetry about each of the axes of $x$ and $y$. 
$\$ 15$. Take the proposition in rectilinear geometry that the diagonals of a parallelogram bisect each other. Let ABCD be a parallelogram in the $\xi \eta$ plane whose diagonals bisect at $\mathrm{E}$. Then in the $x y$ plane (Fig. 2) there correspond to the two parallel lines

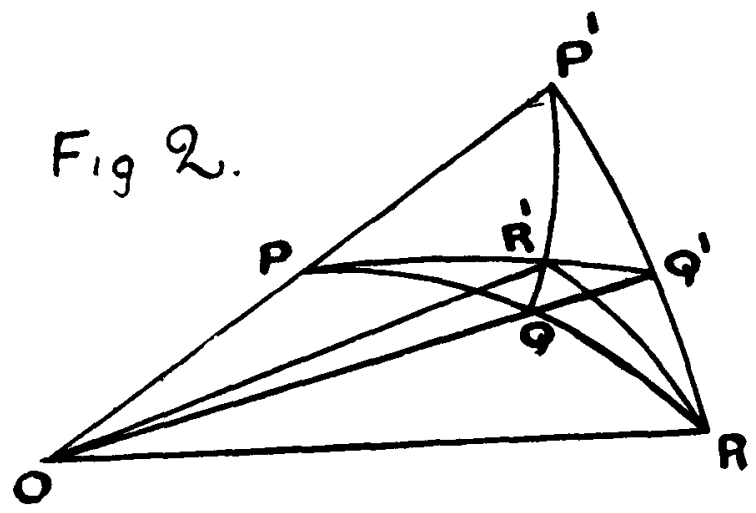

$A B$ and $C D$ two points $P$ and $P^{\prime}$ on the same straight line through the origin; to the two parallel lines $\mathrm{BC}$ and $\mathrm{AD}$ two other points $Q$ and $Q^{\prime}$, also on the same straight line through the origin; to the point $B$ the curve $P Q$;

to the point $\mathrm{D}$ the curve $\mathrm{P}^{\prime} \mathrm{Q}^{\prime}$;

to the line $B D$ the point $R$ at which $P Q$ and $P^{\prime} Q^{\prime}$ intersect;

to the point $A$ the curve $\mathrm{PQ}^{\prime}$;

to the point $C$ the curve $P^{\prime} Q$;

to the line $A C$ the point $R^{\prime}$ at which $P^{\prime}$ and $P^{\prime} Q$ intersect;

to the point $\mathrm{E}$ the curve $\mathrm{RR}^{\prime}$;

and since $E$ is the middle point of $A C$ and of $B D$, therefore $R R^{\prime}$ is the harmonic mean curve between $P Q$ and $P^{\prime} Q^{\prime}$, and also between $\mathrm{PQ}^{\prime}$ and $\mathrm{P}^{\prime} \mathrm{Q}$.

Hence we have the following proposition in curvilinear geometry-if from the origin any two radii vectores be drawn to meet the curve $R P Q$ at $P$ and $Q$ respectively, and the curve $R P^{\prime} Q^{\prime}$ at $P^{\prime}$ and $Q^{\prime}$ respectively, and if the curves $P Q^{\prime}, P^{\prime} Q$ be drawn intersecting at $R^{\prime}$, then the locus of $R^{\prime}$ is the harmonic mean curve to $P Q$ and $P^{\prime} Q^{\prime}$.

Corollary.-If the equation be $\xi x+\eta y=1$, we deduce the harmonic property of the complete parallelogram. 
\$16. Take the proposition that the medians of a triangle are concurrent.

Let $\mathrm{ABC}$ be a triangle in the $\xi \eta$ plane, and let $\mathrm{AD}, \mathrm{BE}$ and $\mathrm{CF}$ be its medians and $K$ their point of intersection.

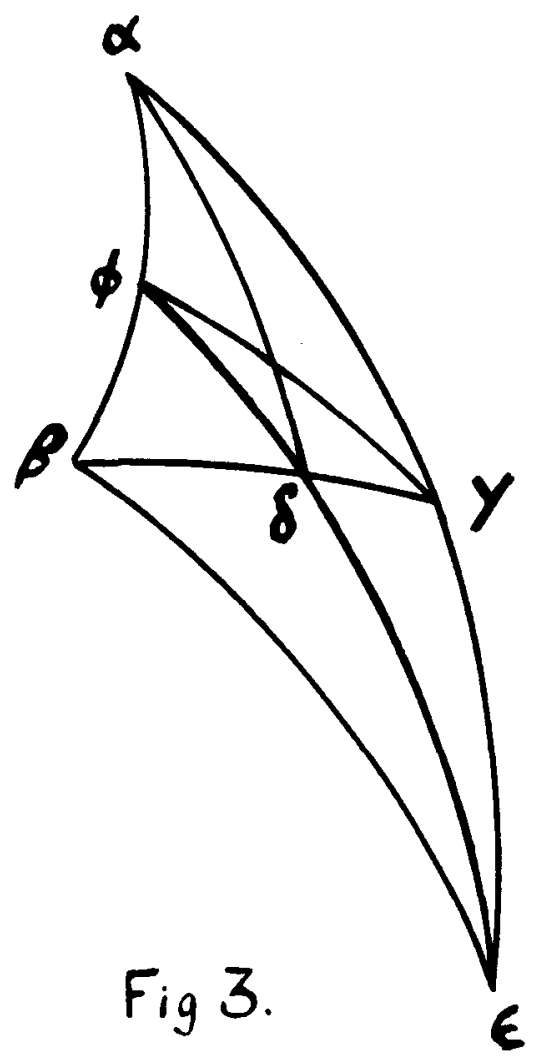

Then in the $x y$ plane (Fig. 3) there corresponds to the triangle $\mathrm{ABC}$ a curvilinear triangle $a \beta \gamma$; to the point $D$ the harmonic mean curve between $a \beta$ and $a \gamma$; to the line AD the point of intersection $\delta$ of this harmonic mean curve with $\beta \gamma$.

Hence we have the proposition that if points $\epsilon$ and $\phi$ be similarly found in $\gamma \alpha$ and $\alpha \beta$, the points $\delta, \epsilon$ and $\phi$ will lie on a curve of the family. 
Corollary.-Putting $\xi x+\eta y=1$ for our equation we get the following proposition (Fig. 4):-If $\mathrm{ABC}$ be a rectilinear triangle

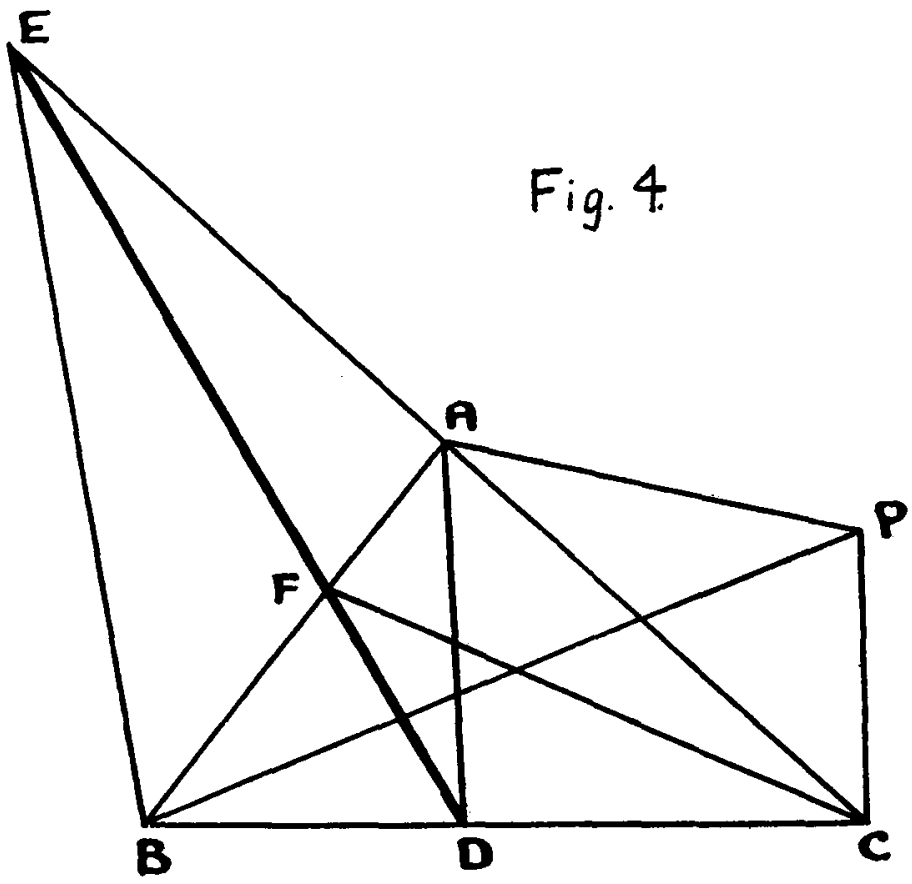

and $P$ any point in its plane ( $P$ being the origin or polar centre), then if $\mathrm{PA}, \mathrm{PB}$ and $\mathrm{PC}$ be joined, and if $\mathrm{AD}$ be drawn cutting $B C$ at $D$ and such that $A(B D C P)$ is a harmonic pencil, and if in the same way points $E$ and $F$ be found in $A C$ and $A B$, then $D, E$ and $\mathrm{F}$ are collinear.

\$17. From the last-mentioned corollary we may deduce the following proposition-if $a \beta \gamma$ be a curvilinear triangle, and if $\pi$ be any other curve of the family cutting $\beta \gamma, \gamma \alpha$ and $a \beta$ at $\delta, \epsilon$ and $\phi$ respectively, and if the points $\delta^{\prime}, \epsilon^{\prime}, \phi^{\prime}$ be taken such that $\beta \delta \delta^{\prime}$, $\gamma \in \alpha \epsilon^{\prime}, \alpha \phi \beta \phi^{\prime}$ are each a harmonic range, then the curves $\alpha \delta^{\prime}, \beta \epsilon^{\prime}$ and $\gamma \phi^{\prime}$ shall be concurrent. 
Corollary. - If $\mathrm{ABC}$ be a rectilinear triangle (Fig. 5), and if any straight line cut its sides at $\mathrm{D}, \mathrm{E}$ and $\mathrm{F}$ respectively, and if $\mathrm{D}^{\prime}, \mathrm{E}^{\prime}$, and $\mathrm{F}^{\prime}$ be the harmonic conjugates of $\mathrm{D}, \mathrm{E}$ and $\mathrm{F}$ with respect to $\mathrm{BC}, \mathrm{CA}$ and $\mathrm{AB}$ respectively, then $\mathrm{AD}^{\prime}, \mathrm{BE}^{\prime}$ and $\mathrm{CF}^{\prime}$ shall be concurrent at $K$.

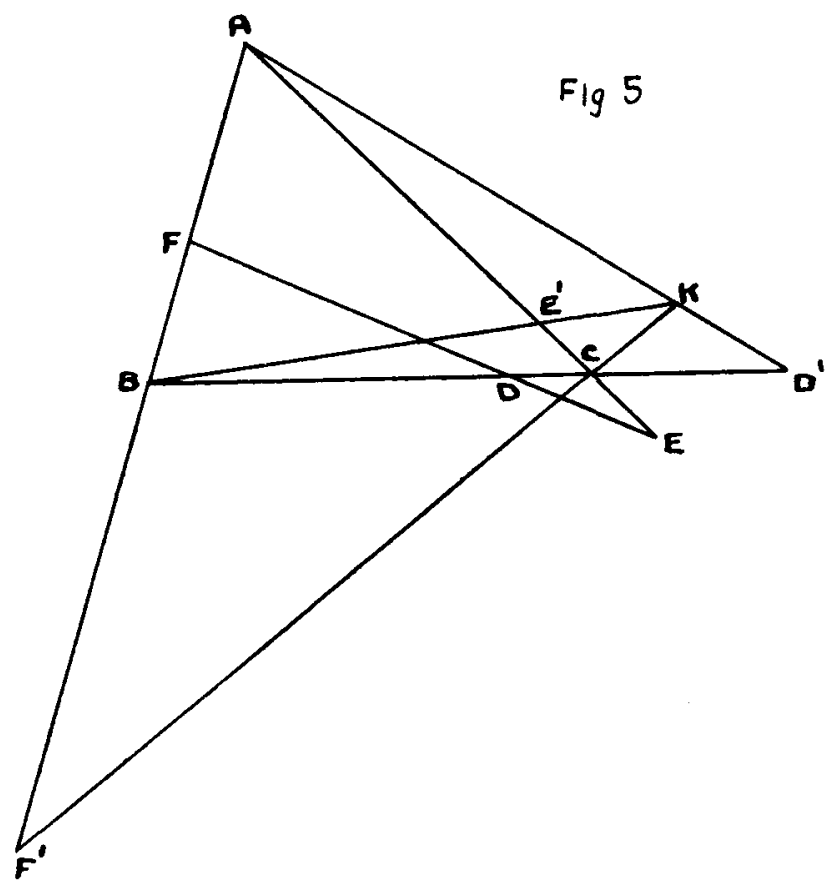

$\$ 18$. Take the proposition that the perpendiculars in a rectilinear triangle are concurrent. We shall consider the case only where $(-1)^{n}=-1$.

Let $\mathrm{ABC}$ (Fig. 6) be a triangle in the $\xi \eta$ plane, and let $\mathrm{AD}, \mathrm{BE}$ and $\mathrm{CF}$ be its perpendiculars meeting at $\mathrm{K}$.

Then in the $x y$ plane there corresponds (Fig. 7) to the triangle $A B C$ a curvilinear triangle $\alpha \beta \gamma$. Join $O \alpha$ and draw $\mathrm{Oa}^{\prime}$ perpendicular to $\mathrm{O} a$. Then every line in the $\xi \eta$ plane parallel to BC is represented by a point on $\mathrm{O} \alpha$ and every line perpendicular to $\mathrm{BC}$ by a point on $\mathrm{Oa}^{\prime}$. The point where $\mathrm{Oa}$ meets $\beta \gamma$ will represent the line perpendicular to $\mathrm{BC}$ passing through $\mathrm{A}$, that is, 
the perpendicular AD. Call this point $a^{\prime}$. Then if the points $\beta^{\prime}$ and $\gamma^{\prime}$ on $\alpha \gamma$ and $\alpha \beta$ respectively which represent $\mathrm{BE}$ and $\mathrm{CF}$ be found, the points $\alpha^{\prime}, \beta^{\prime}$ and $\gamma^{\prime}$ will lie on a curve of the family.

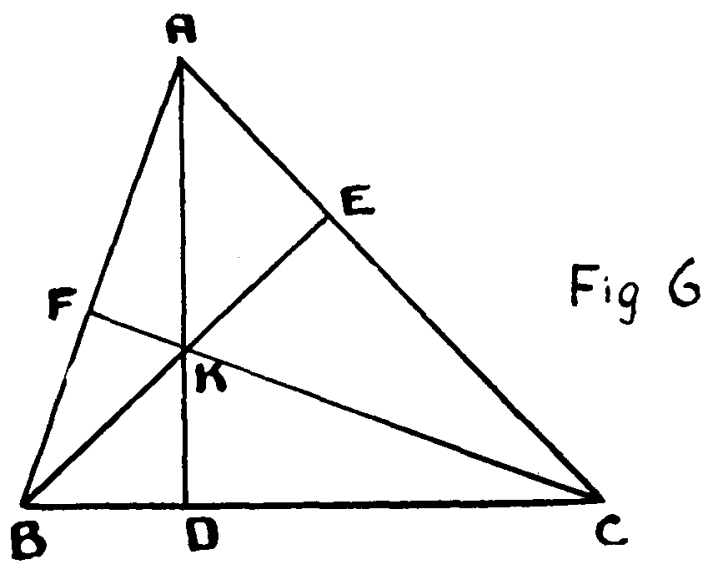

The symmetry of this figure will be noticed. If we take any three of the four lines $\alpha \beta, \beta \gamma, \gamma \alpha$ and $\alpha^{\prime} \beta^{\prime} \gamma^{\prime}$ as the initial triangle, and find the curve passing through the feet of the perpendiculars constructed as in the figure, that curve will be the remaining line of the four; this corresponds to the proposition that if we take the triangle formed by any three of the points $\mathrm{A}, \mathrm{B}, \mathrm{C}, \mathrm{H}$, its perpendiculars will intersect at the fourth of these points.

Corollary (1).-If $\xi x+\eta y=1$ be the equation, we have the following proposition (Fig. 8):-If $\mathrm{ABC}$ be a rectilinear triangle and $P$ any point on its plane, and if $\mathrm{PD}, \mathrm{PE}, \mathrm{PF}$ be drawn perpendicular to $\mathrm{PA}, \mathrm{PB}, \mathrm{PC}$ respectively, meeting $\mathrm{BC}, \mathrm{CA}$ and $\mathrm{AB}$ at $\mathrm{D}, \mathrm{E}$ and $\mathrm{F}$ respectively, then $\mathrm{DEF}$ is a straight line. It will be noticed that if any three of the four lines $\mathrm{AB}, \mathrm{BC}, \mathrm{CA}$ and $\mathrm{DE}$ be taken as the sides of the triangle, then the construction above described with regard to the point $P$ will give us the fourth of these lines.

Corollary (2). -The circles described on the three diameters of a complete quadrilateral meet at a point. 
$\S 19$. Starting from corollary 1, as a proposition in the $\xi \eta$ plane we may deduce a corresponding proposition in curve geometry in the $x y$ plane when $(-1)^{n}=-1$.

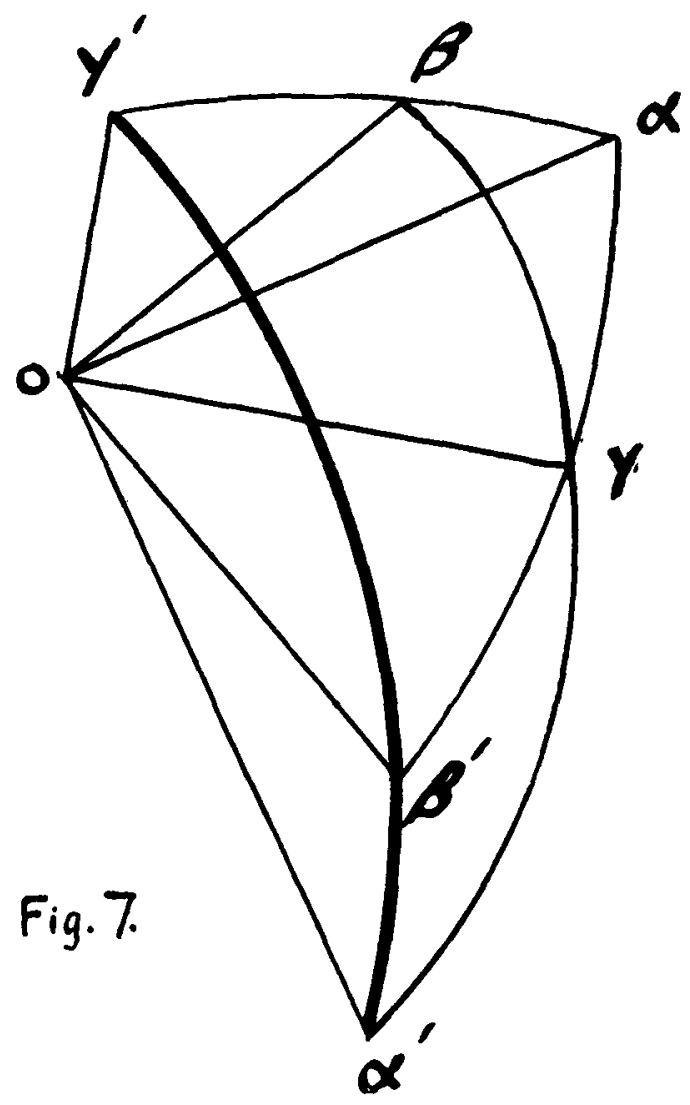

There correspond in the $x y$ plane (Fig. 9) to the triangle ABC a curvilinear triangle $\alpha \beta \gamma$; to the point $\mathrm{P}$ a curve $\pi$; to $\mathrm{PA}, \mathrm{PB}$ and $P C$ the points $\alpha^{\prime}, \beta^{\prime}, \gamma^{\prime}$, at which $\pi$ meets $\beta \gamma, \gamma \alpha$ and $\alpha \beta$ respectively; to $\mathrm{PD}, \mathrm{PE}$ and $\mathrm{PF}$ the points at which the straight lines $O a^{\prime \prime}, O \beta^{\prime \prime}, O \gamma^{\prime \prime}$ drawn perpendicular to $O a^{\prime}, O \beta^{\prime}$ and $O \gamma^{\prime}$ meet the curve $\pi$; call these points $\alpha^{\prime \prime}, \beta^{\prime \prime}, \gamma^{\prime \prime}$; to the points $\mathbf{D}, \mathbf{E}$ and $\mathbf{F}$ the curves $a a^{\prime \prime}, \beta \beta^{\prime \prime}, \gamma \gamma^{\prime \prime}$. And since $\mathrm{D}, \mathrm{E}$ and $\mathrm{F}$ are collinear we have proved that $a a^{\prime \prime}, \beta \beta^{\prime \prime}$ and $\gamma \gamma^{\prime \prime}$ are concurrent say at $K$. 
31
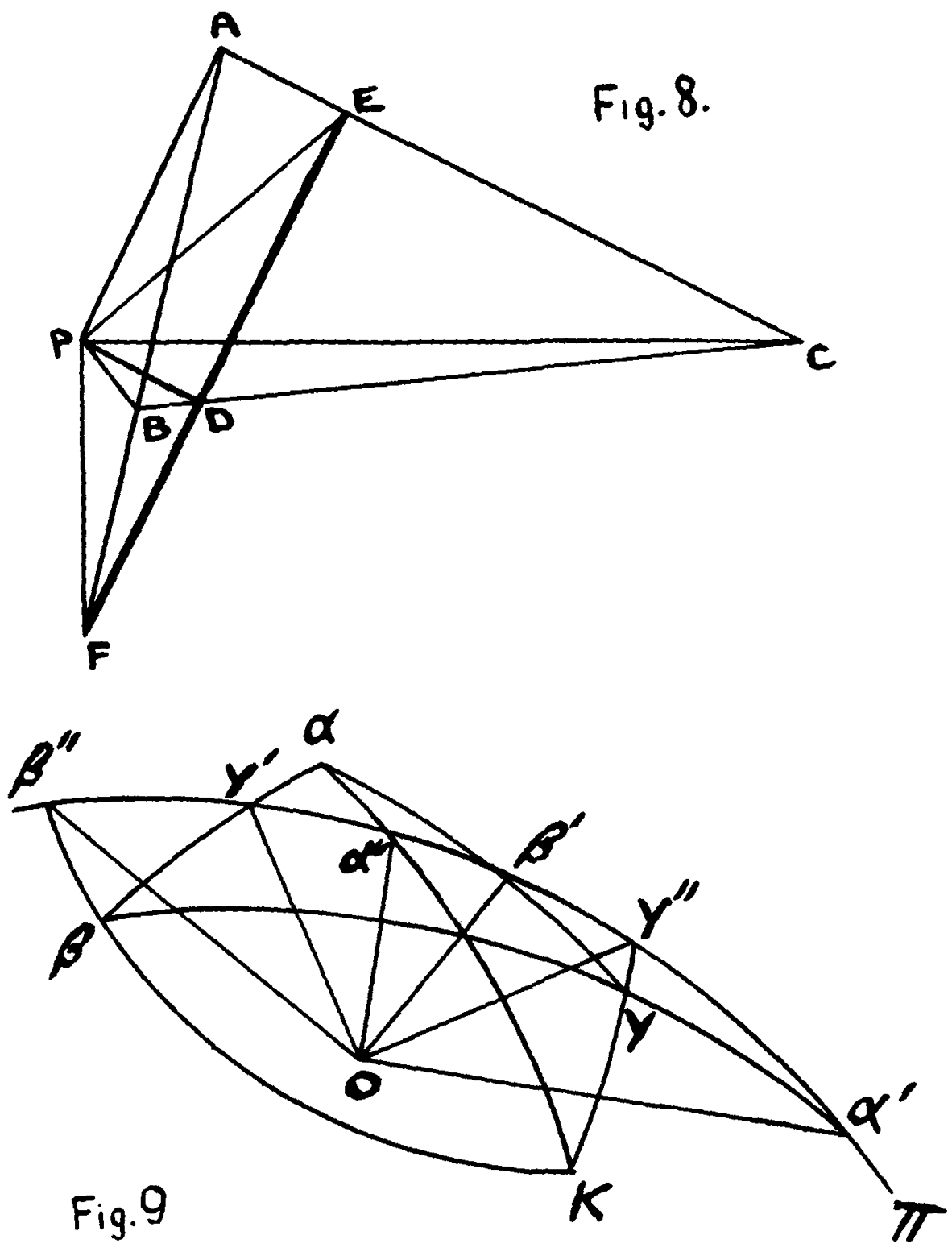

https://doi.org/10.1017/S0013091500033769 Published online by Cambridge University Press 
Corollary.-If we take $\xi x+\eta y=1$ as the equation, we have the following proposition (Fig. 10):-If any straight line meet the sides of a rectilinear triangle $A B C$ at $A^{\prime}, B^{\prime}$ and $C^{\prime}$ respectively, and if from any point $\mathrm{P}, \mathrm{PA}^{\prime \prime}, \mathrm{PB}^{\prime \prime}, \mathrm{PC}^{\prime \prime}$ be drawn respectively perpendicular to $\mathrm{PA}^{\prime}, \mathrm{PB}^{\prime}, \mathrm{PC}^{\prime}$ and meeting the given transversal at $\mathrm{A}^{\prime \prime}, \mathrm{B}^{\prime \prime}, \mathrm{C}^{\prime \prime}$, then $\mathrm{AA}^{\prime \prime}, \mathrm{BB}^{\prime \prime}$ and $\mathrm{CC}^{\prime \prime}$ will be concurrent at a point $\mathrm{P}^{\prime}$.

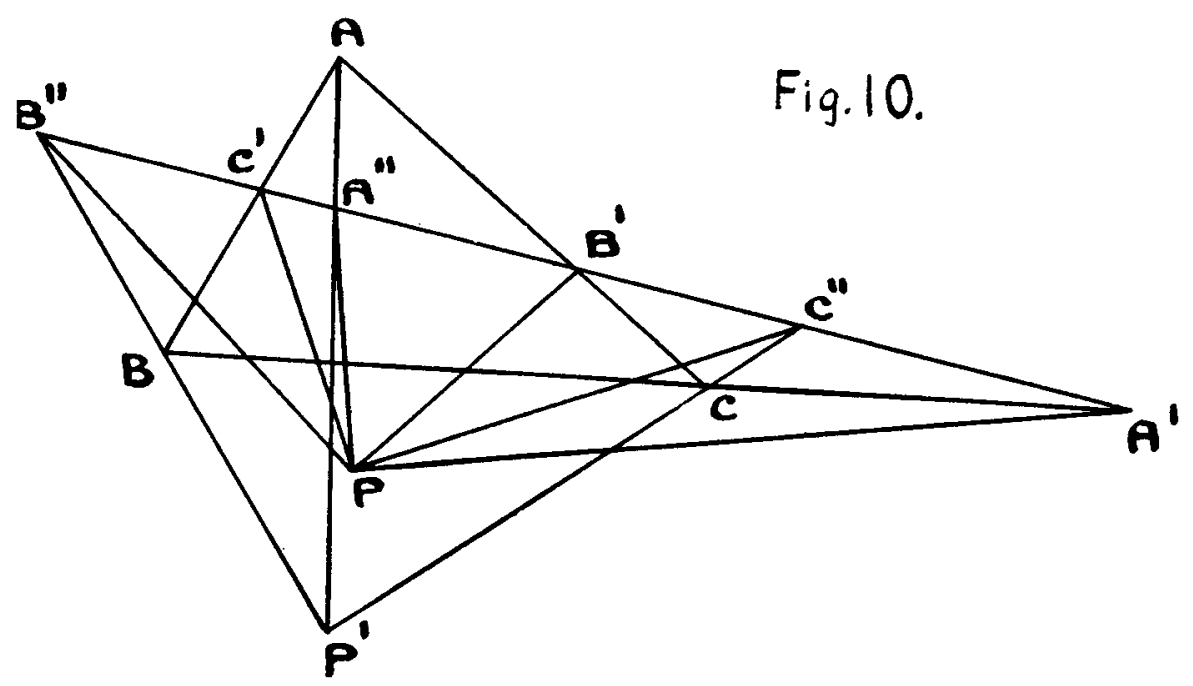

$\$ 20$. Starting from the corollary in the $\xi \eta$ plane we get the following proposition in the $x y$ plane, where $(-1)^{n}=-1:-$ If the curves $\kappa a, \kappa \beta$ and $\kappa \gamma$ be drawn joining any point $\kappa$ to the vertices $\alpha, \beta, \gamma$ of a curvilinear triangle $\alpha \beta \gamma$, and if any curve $\pi$ meet $\kappa \alpha, \kappa \beta$ and $\kappa \gamma$ at $\alpha^{\prime}, \beta^{\prime}$ and $\gamma^{\prime}$ respectively, if the origin $O$ be joined to $a^{\prime}, \beta^{\prime}$ and $\gamma^{\prime}$, and if $O a^{\prime \prime}, O \beta^{\prime \prime}, O \gamma^{\prime \prime}$ be drawn perpendicular to $O a^{\prime}, O \beta^{\prime}$ and $O \gamma^{\prime}$ respectively, meeting the curve a at $\alpha^{\prime \prime}, \beta^{\prime \prime}, \gamma^{\prime \prime}$, and if the curves $\kappa \alpha^{\prime \prime}, \kappa \beta^{\prime \prime}$ and $\kappa \gamma^{\prime \prime}$ meet $\beta \gamma, \gamma \alpha$ and $\alpha \beta$ at $\alpha^{\prime \prime \prime}, \beta^{\prime \prime \prime}$, and $\gamma^{\prime \prime \prime}$ respectively, then will $\alpha^{\prime \prime \prime}, \beta^{\prime \prime \prime}$, and $\gamma^{\prime \prime \prime}$ lie on a curve of the family. 
The corollary in straight lines is-if $\mathrm{ABC}$ is a rectilinear triangle, and if any point $\mathrm{K}$ be joined to $\mathrm{A}, \mathrm{B}$ and $\mathrm{C}$, and if $\mathrm{A}^{\prime}, \mathrm{B}^{\prime}, \mathrm{C}^{\prime}$ be the points at which any transversal cuts $\mathrm{KA}, \mathrm{KB}$ and $\mathrm{KC}$ respectively, then if $\mathrm{P}$ be any other point, and if $\mathrm{PA}^{\prime \prime}, \mathrm{PB}^{\prime \prime}, \mathrm{PC}^{\prime \prime}$ be drawn perpendicular to $\mathrm{PA}^{\prime}, \mathrm{PB}^{\prime}$ and $\mathrm{PC}^{\prime}$ respectively, meeting the transversal at $A^{\prime \prime}, B^{\prime \prime}$ and $C^{\prime \prime}$, and if $\mathrm{AA}^{\prime \prime}, \mathrm{BB}^{\prime \prime}, \mathrm{CC}^{\prime \prime}$ cut $\mathrm{BC}, \mathrm{CA}$ and $\mathrm{AB}$ at $\mathrm{A}^{\prime \prime \prime}, \mathrm{B}^{\prime \prime \prime}$ and $\mathrm{C}^{\prime \prime \prime}$ respectively, then will $\mathrm{A}^{\prime \prime \prime} \mathrm{B}^{\prime \prime \prime} \mathrm{C}^{\prime \prime \prime}$ be a straight line.

This corollary might again be used as the basis for a fresh proposition, and so on indefinitely.

$\$ 21$. Take the proposition in rectilinear geometry that if two vertices of a triangle move on a fixed straight line, while the three sides pass through three collinear points, the locus of the third vertex is a straight line, and that if two sides of a triangle pass through fixed points while the vertices move on three concurrent lines, the third side will pass through a fixed point.

The corresponding proposition is-if two sides of a curvilinear triangle pass through fixed points while the vertices move on three concurrent curves of the same family, the third side will pass through a fixed point, and if two vertices move on curves of the family, while the three sides pass through three fixed points lying on a curve of the family, the third vertex will also lie on a curve of the family.

\$2. Take the proposition that the locus of the middle points of the intercepts on a series of parallel straight lines by two fixed straight lines is a straight line passing through their point of intersection.

The corresponding proposition in curvilinear geometry is-if the point of intersection of two curves of the family passing respectively through two fixed points lies on a straight line through the origin, then their harmonic mean curve will always pass through a fixed point lying on the same curve of the family as the two given fixed points.

Further, the two given fixed points, the fixed point through which the harmonic mean passes, and the point of intersection of the given line through the origin with the curve passing through the two given fixed points, will form a harmonic range. 


\section{4}

$\$ 23$. It is evident that these examples might be extended in number almost indefinitely.

\$24. Further, it is plain that the method might be extended to the geometry of three dimensions. The same reasoning could be applied to the equations

$$
\begin{aligned}
& f(\xi, \eta, \zeta, x, y, z)=0, \\
& \xi x^{n}+\eta y^{n}+\zeta z^{n}=1, \\
& \xi x+\eta y+\zeta z=1, \text { etc., }
\end{aligned}
$$

as to the corresponding equations in two dimensions. 